\title{
Video techniques and data compared with observation in emergency trauma care
}

\author{
C F Mackenzie, Y Xiao
}

Qual Saf Health Care 2003;12(Suppl II):ii51-ii57

Video recording is underused in improving patient safety and understanding performance shaping factors in patient care. We report our experience of using video recording techniques in a trauma centre, including how to gain cooperation of clinicians for video recording of their workplace performance, identify strengths of video compared with observation, and suggest processes for consent and maintenance of confidentiality of video records. Video records are a rich source of data for documenting clinician performance which reveal safety and systems issues not identified by observation.

Emergency procedures and video records of critical events identified patient safety, clinical, quality assurance, systems failures, and ergonomic issues. Video recording is a powerful feedback and training tool and provides a reusable record of events that can be repeatedly reviewed and used as research data. It allows expanded analyses of time critical events, trauma resuscitation, anaesthesia, and surgical tasks. To overcome some of the key obstacles in deploying video recording techniques, researchers should (1) develop trust with video recorded subjects, (2) obtain clinician participation for introduction of a new protocol or line of investigation, (3) report aggregated video recorded data and use clinician reviews for feedback on covert processes and cognitive analyses, and (4) involve multidisciplinary experts in medicine and nursing.

See end of article for authors' affiliations

Correspondence to Dr C F Mackenzie, The Charles McC Mathias Jr, National Study Centre for Trauma and EMS, $701 \mathrm{~W}$ Pratt Street, 518 Baltimore, MD 21201-1023, USA; cmack003@ umaryland.edu
A $\mathrm{n}$ understanding of the strengths and weaknesses of human performance has direct implications for strategies to improve quality of health care and patient safety. However, our knowledge of human performance in real, complex, and dynamic environments such as those found in clinical care settings is limited. ${ }^{1}$ Studies of healthcare providers in their natural settings could provide an insight into how teams work under time pressure, with constant interruptions, and in suboptimal workplaces. Previous studies of naturalistic decision making and team performance have highlighted the advantages of understanding expert human activities in real, complex, and dynamic environments. ${ }^{2}$ Primary strengths of ethnographic observations are that they support a discovery process, ${ }^{3}$ draw attention to significant phenomena, and suggest new theories whose validity and generality can then be evaluated through additional studies. ${ }^{4}$ In comparison, studies carried out in laboratory settings do not recreate many of the real life variables such as risk, uncertainty, composition of teams, and the workplace domain. These are significant factors in determining performance during real world dynamic and stressful events.

This paper describes our experiences of using video recording in research in a trauma centre during real emergency resuscitation, surgery, and anaesthesia; it is not about technical aspects of video recording. The goal is to summarize the lessons learned and principles used in deploying video recording methodologies and in exploiting the advantages offered by the video medium. ${ }^{56}$ Video is perceived as the richest medium for capturing the smallest and briefest particulars of human interaction while retaining the context of the event and making it available for analyses by multiple or independent subject matter experts. ${ }^{5}$ Video recording in the medical environment makes it possible for clinicians to review their own activities and for analysts to extract qualitative and quantitative data.

Despite these advantages of video recording, challenges abound-such as gaining support from those being recorded, securing patient confidentiality, overcoming medicolegal obstacles, and effectively using the medium. ${ }^{56}$ This paper focuses on three key methodology issues relating to the use of video techniques in clinical environments:

- the importance of communication with, and development of trust with, video recorded subjects;

- the differing use and content of information available from video compared with observational data;

- suggestions to assist issues of participant consent as well as to minimize loss of confidentiality/privacy.

\section{COMMUNICATION WITH SUBJECTS TO GAIN THEIR PARTICIPATION IN VIDEO RECORDING}

Development of trust with video recorded subject

Our work was based in a single trauma centre at the University of Maryland. Details of this facility are shown in box 1 .

The purpose of our original video recording effort was to examine real events in detail and to detect how clinicians performed in stressful conditions of trauma patient resuscitation. Because of medicolegal, employment, privacy, 
Box 1 University of Maryland shock trauma centre

- Patients: more than $7000 /$ year

- Motor vehicle crashes $55 \%$, interpersonal violence $16 \%$, falls $21 \%$, other $18 \%$

- $42 \%$ admitted directly by helicopter from scene of injury

- $85 \%$ within 1 hour ("golden hour") directly from scene of injury

- Facilities: stand alone 110 bed trauma centre

- Ten resuscitation bays, six operating rooms, seven PACU beds, trauma critical care unit, neurotrauma unit, intensive care

- Staffing: faculty surgeons, anaesthesiologists, emergency medicine, critical care physicians, fellows, residents, nurse anaesthetists, trauma nurses, technicians, administrators

and confidentiality issues and review of their own performance by peers, many clinicians were wary about video recording. We used several procedures to gain the confidence and trust of the research subjects that video recording would only be used for research and educational purposes.

Control of video recording was given to the study research subjects (initially this was anaesthesiology clinical care providers). They started and stopped the equipment, which was located within arms' reach of their normal working position at the head of the patient (fig 1). LED lights on the camera and video cassette recorder were illuminated during recording so that other clinicians working in the same area as the research subjects could be made aware that video recording was underway. Collecting of video data occurred in only two locations among 10 in the resuscitation areas and two of six operating rooms. This enabled those who felt uncomfortable with the video recording to have an alternative location for providing patient care. Video recording was focused around a specific stressful task (airway management) in association with a specific research protocol. The video record was available for review after the event and, on request of the video recorded subject(s), it would be erased if they considered that inappropriate or liability issues were recorded.

The phases of different strategies for video acquisition are shown in box 2 .

\section{Phased approach to scale up of video recording}

For the first study video cassette recorders and single ceiling mounted cameras installed in two trauma resuscitation unit bays and two operating rooms were focused only on the activities of the anaesthesia care providers. The advantage of this approach was that the equipment was relatively inexpensive and the collaboration of only one group of clinicians was required. The process of video acquisition was fully automated, with the equipment located on the anaesthesia machine. A single button pushed by the research subject started recording of audio-video signals, the machine readable time code, and video overlay of vital signs data through a serial interface and video card (fig l).

For the second upgrade in video acquisition we expanded the recording site locations to six of 10 trauma resuscitation unit bays and added pan-tilt zoom cameras. We found that in some of the earlier video records, the single camera view was obstructed by equipment or other care providers. The additional and more detailed view was synchronized by digital time code with the environmental overview camera. Having shown that no adverse consequences occurred during the earlier research subject controlled video recording, a telecontrol centre was built to integrate all the audio-video and vital signs signals where a technician instigated video recording remotely (fig 2). A mobile video acquisition system with a tethered head mounted camera was designed to be useable in all the trauma resuscitation unit bays or operating rooms not already instrumented for video acquisition (fig 3 ).

The third upgrade of video acquisition capability included instrumenting all the trauma resuscitation unit bays and six operating rooms with multiple ceiling mounted cameras. ${ }^{13}$ In addition, a wireless head mounted camera was designed and built to allow image retrieval from operators during emergency tasks (fig 4). An infrared mobile wireless audio

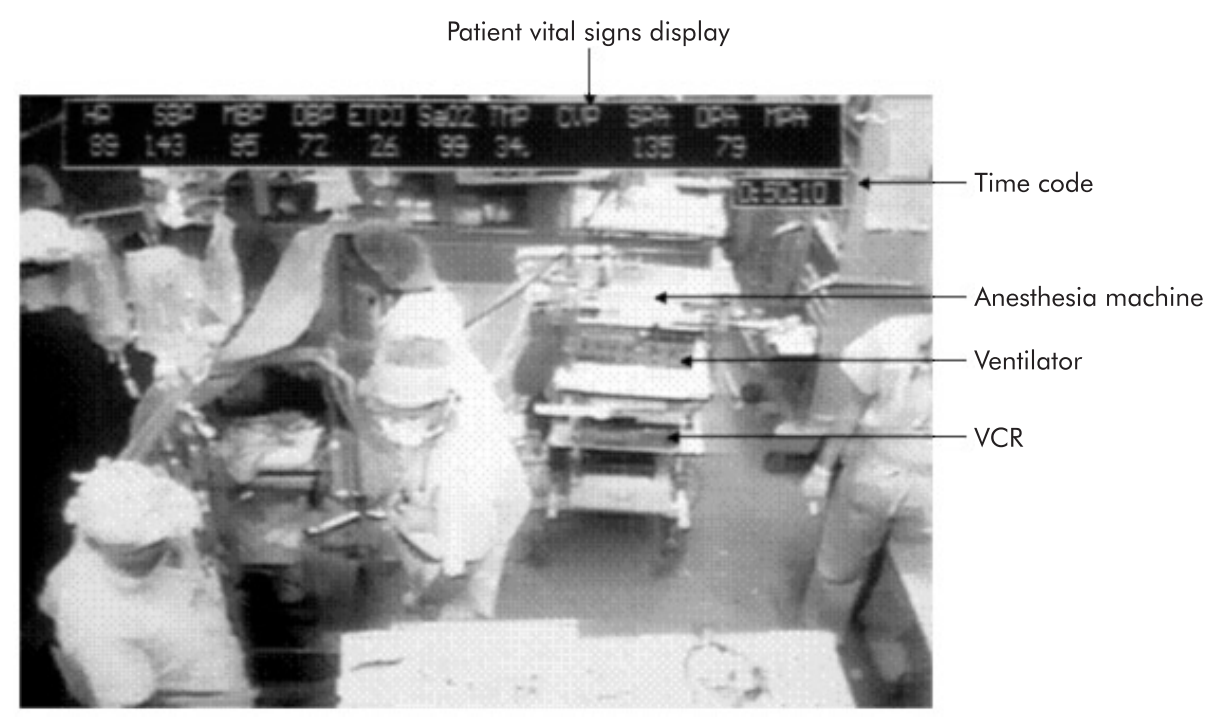

Figure 1 Operating room activity with overlay of patient vital signs: $\mathrm{HR}=$ heart rate; $\mathrm{SBP}, \mathrm{MBP}, \mathrm{DBP}=$ systolic, mean, and diastolic arterial pressure; $\mathrm{ETCO}=$ end tidal $\mathrm{CO}_{2}$ pressure; $\mathrm{SaO}_{2}=\mathrm{O}_{2}$ saturation; $\mathrm{TMP}=$ temperature $\left({ }^{\circ} \mathrm{C}\right) ; \mathrm{SPA}, \mathrm{DPA}=$ non-invasive blood pressure or pulmonary artery pressure when used. Time code is shown at the bottom right of the vital signs display. The videocassette recorder (VCR) is seen two shelves from the bottom of the anesthetic machine/ventilator module. Facial features are blurred. 


\section{Box 2 Strategies for video acquisition}

Phase I (1991-1996)

- Audio video recording capability in four locations (2/ 10 resuscitation bays, 2/6 operating rooms), 120 recordings made. Reports are available on performance and "Decision making under stress in trauma patient resuscitation" at http://hfrp.umm.edu and "Remote decision making: media, cues and diagnosis during dynamic tasks" at hitp://hfrp.umm.edu.

- Equipment used shown in fig 1.

Phase II (1996-1998)

- Telecontrol centre for remote video recording (fig 2). Six pan-tilt-zoom cameras and environmental cameras installed in resuscitation bays, tethered head camera (fig 3), mobile environmental camera and patient vital signs monitor interface allowed video recording in all operating rooms and critical care sites. Integration of four images and video analysis in telecontrol centre.

Phase III (1998-2003)

- All 10 resuscitation bays instrumented with pan-illtzoom environmental cameras and patient vital signs interfaces. Head mounted wireless video camera (fig 4) was developed to be worn by an operator (such as a traumatologist during chest tube insertion). All six operating rooms cabled with vital signs interface and cameras. Fourth task specific camera installed at back of each of 10 resuscitation bags to obtain details of emergency and elective invasive procedures.

head set allowed remotely situated researchers (in the telecontrol centre) to query the operators or clinicians at appropriate intervals during video acquisition.

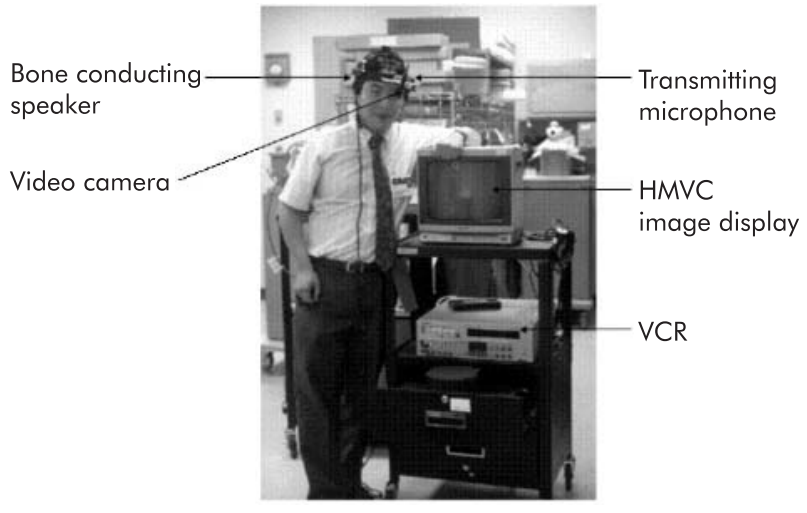

Figure 3 Portable video recording system containing a video cassette recorder (VCR) and a head mounted tethered video camera with duplex audio to allow remote teleconsultation using the video image and voice. A modification of this system included a second environmental camera attached to the display monitor, a patient vital signs interface, and a second VCR to record both the head camera and the environmental camera with the images displayed remotely as picture-in-picture.

Together with the equipment scale up, we were able to recruit other clinicians to join an ever expanding group of studies that subsequently involved surgeons, nurses, anaesthesiologists, emergency medicine physicians, and prehospital providers. The most recent upgrade is that we have instrumented all 19 new operating rooms (dubbed the "operating rooms of the future") at the University of Maryland Medical System with an audio-video acquisition system and telecontrol centre for coordination of the operating rooms.

\section{Introduction of new protocol}

When the first protocol involving video recording was to be introduced, the following strategy was employed to facilitate clinician acceptance. An "open house" was arranged showing typical views and marking the location in the trauma resuscitation unit that would be included in the video image. Several meetings were held at which one-on-one discussions

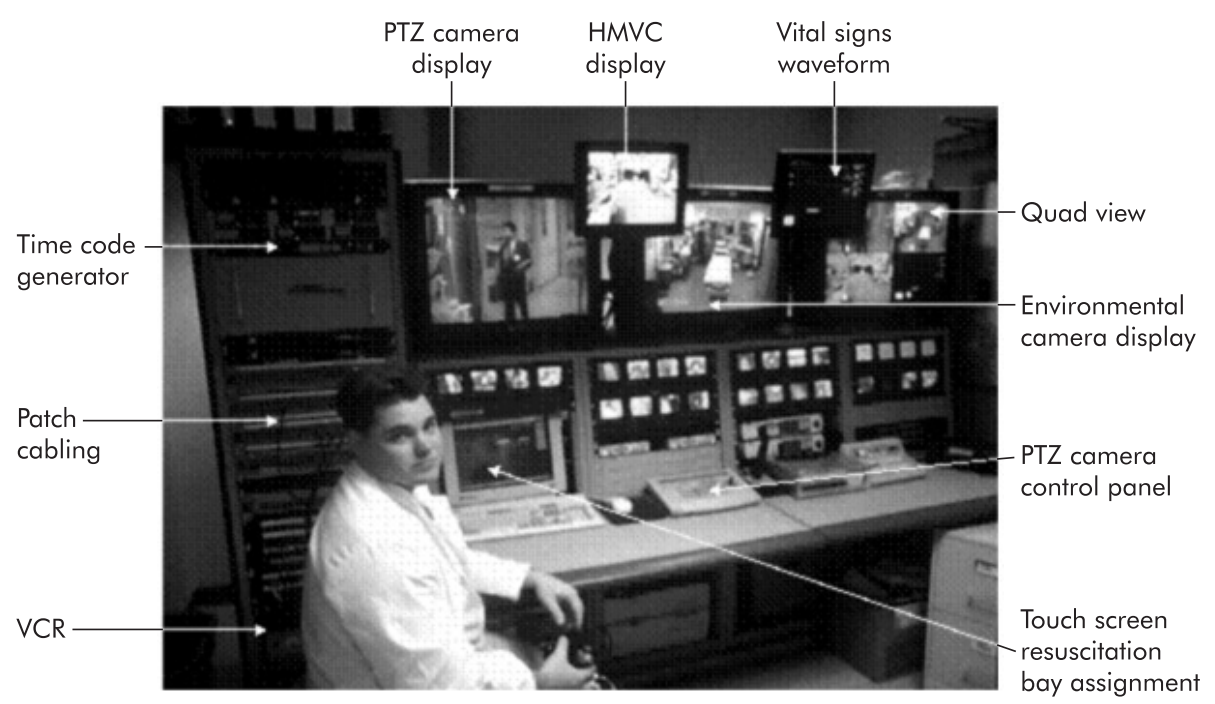

Figure 2 Telecontrol centre showing three full screen displays above 28 small screen individual camera images. The right full screen displays the "quad" image. The two stand out screens between the full screen monitors display the vital sign waveform data (on right) and the wireless head camera image (on left). The touch screen panel (above keyboard) allows the video technician to display any one of the video instrumented locations with a single key touch. The time code generator and patch board is shown to the left of the video technician. Four video cassette recording machines (VCR) are arranged in the rack beneath the video patch board. 


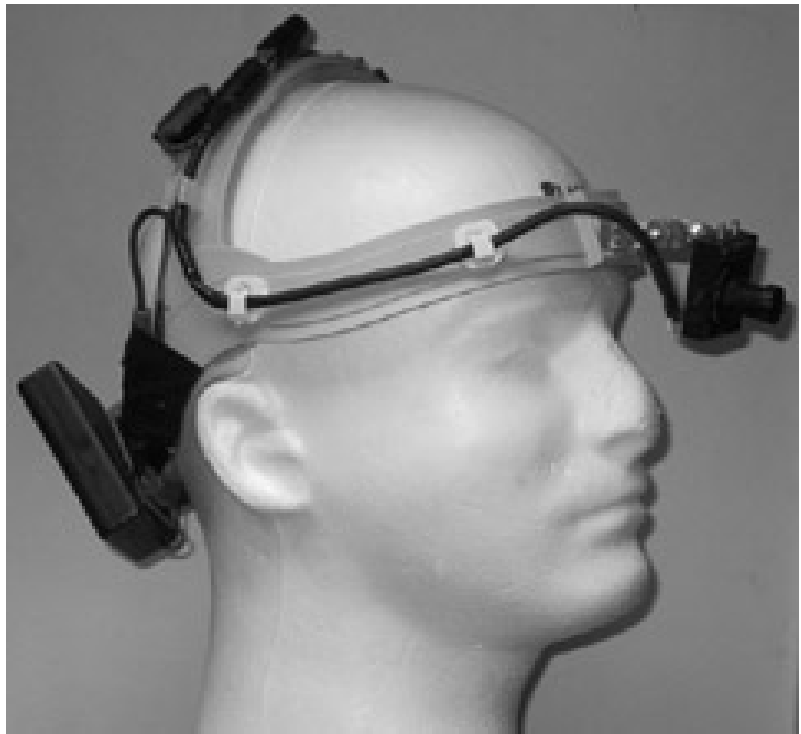

Figure 4 The wireless head camera had four AA batteries in a box mounted over the occiput. The camera was on a swivel mount between the eyes. The video transmitter was positioned in the middle of the 10 resuscitation bay trauma resuscitation unit layout and allowed free range movement with image transmission over about 100 feet.

occurred with night and day shift nursing staff. Medical staff meetings were also arranged for surgeons, anaesthesiologists, and orthopaedists. The most frequently asked questions were then discussed at a weekly multidisciplinary staff meeting (medical/nursing/technicians) and all questions were answered in both group settings and one-on-one informal meetings. Protocols for specific video recording research were planned with all the major stakeholders. Protection of human subjects (both patients and care providers) was secured through a formal approval process by the Institutional Review Board (IRB). Consent forms were distributed and discussed extensively with the research subjects who would be video recorded. An approach for information sharing about video recording is shown in fig 5 .

\section{Feedback from video analysis}

Clinicians became supportive of video recording when they could see that participation was beneficial. Video recording was also used as part of the quality management program of potential benefit for all care providers-for example, were they carrying out universal precautions to protect themselves against blood borne infections and could we reduce staff injury by needle sticks and knife blade injuries by data from video analysis? Analyses of video records were used to develop best practices for brief, risky, but beneficial tasks such as tracheal intubation, chest tube insertion, subclavian vein catheterization. ${ }^{78}$ We helped the nursing staff in the trauma resuscitation unit to redesign the nurses' supply cart, resulting in a more efficient supply and restocking process. We redesigned the anaesthesia workspace in the trauma resuscitation unit, as it was previously restricted and limited in access to patient monitors and equipment such as oxygen supply and suction systems that are vital to effective emergency management of trauma patients. ${ }^{9}$ The ergonomic analysis of chest tube insertions from video records identified major improvements in the content of the instrument tray and surgical techniques for tube thoracostomy. ${ }^{10}$

The importance of having the support of the video recorded subjects who functioned as subject matter experts (SME) cannot be overstated. Their collaboration was essential to detecting the covert processes, cognitive analyses, and to

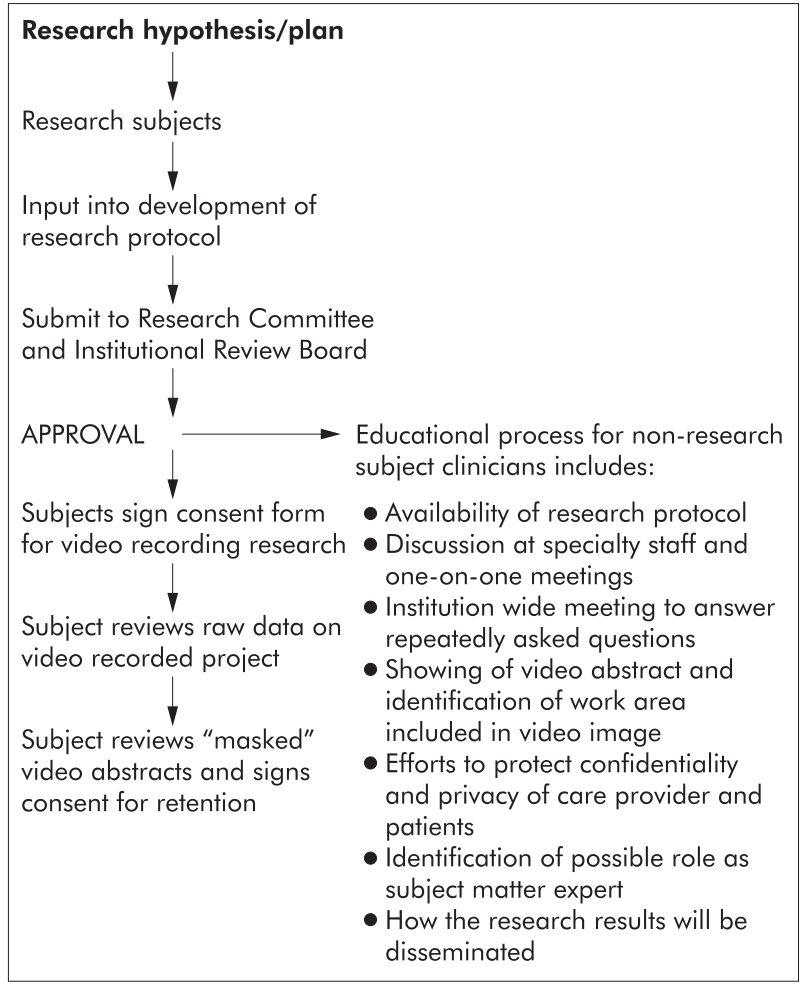

Figure 5 Approach for information sharing about video recording.

clarify ambiguous situations or communications. ${ }^{11}$ Some of these same video records were then used after review by the SME to examine remote decision making, media, cues, and diagnosis during dynamic tasks. ${ }^{12}$ The advantage of SME review is that the process provides both feedback to SME clinicians and results in an additional source of data interpretation.

\section{DIFFERING USE AND CONTENT OF VIDEO COMPARED WITH OBSERVATION Information acquisition}

Observational data collection is an active process that requires the presence of a trained observer (or observers) concurrent with the occurrence of the events of interest. A conceptual framework with "a priori" intent guides data collection during observation. Data parsing inevitably occurs, and fleeting events, simultaneous interventions or brief communications are very difficult to observe and document accurately, ${ }^{3}$ whereas video passively acquires audio and image data in a reusable record. Miniaturization of video equipment allows unobtrusive ceiling mounted cameras to record unobstructed "bird's eye" views not available to standing observers. Video records are raw continuous data whereas observational data only collect information noted by the observer. ${ }^{13}$

Although observational field studies have made valuable contributions and are the traditional ethnographic approach to identifying sources of performance problems and opportunities for improvement, they lack verifiable data and systematic feedback of images for review by participants. ${ }^{13}$ Video recording in the medical environment makes it possible for clinicians to review their own activities and for analysts to extract qualitative and quantitative data. ${ }^{13}$ If communication occurs among team members, cognitive aspects of the task may be revealed. However, the behavior of skilled problem solvers may not reveal the underlying mental processes and 
the expert often finds it difficult to articulate the processes by which a solution is obtained. ${ }^{14}$ The video record may be useful as stimulus material to allow the expert to identifiably reveal his/her expertise. We found that observations and interviews were useful knowledge elicitation techniques in preparation for and after video recording, but the results were often unwieldy and difficult to interpret when complex dynamic tasks were performed by experts in their real workplace. ${ }^{15}$

\section{Data analysis from video records compared with observation}

In contrast to a video record, applications of observational studies are more exploratory. The subtleties of body language and eye movements and fleeting utterances captured by video are only able to be included in theories or conceptual frameworks generated by observation. Observation provides a realistic view of the complexity of the work environment and can be used to develop empirically grounded hypotheses. ${ }^{3}$ Observation allows domain experts to uncover collaborative demands and strategies that practitioners have developed in response to those demands. Observations can show how existing artefacts are used to support such demands. In contrast to other scientific methods, observation is focused on discovery rather than hypothesis testing. ${ }^{3}$ Ambiguous communications central to design interactions cannot be recorded by observation in context as occurs with video. ${ }^{16}$ In comparison, multiple domain experts and the video recorded subjects can themselves repeatedly review the raw video record after the event. Fine grained analysis can include all the nuances of contextual and systems factors because second-by-second behavioral and verbal interactions are recorded with video. Video recording also allows expanded analysis of time critical brief or uncertain events by repeated replays or even frame by frame analysis.

\section{Generalizable findings about video recording}

We found the principal and most useful method of video acquisition was to obtain video clips of short duration (515 minutes) as these provided a rich and ample source of data. The video clips were taken in the context of dynamic portions of patient care-for example, initial reception and resuscitation of a multiply traumatized patient—or particular tasks that were beneficial but risky-for example, airway management, chest tube insertion for hemo or pneumothorax-or those with a high incidence of complication-for example, subclavian central venous line insertion.

When clinical events that have a specific start and defined end time are video recorded, the video recording and its subsequent analysis have more focus; the data are more rapidly extracted and produce more significant findings than continuous recording to capture random events. Task oriented video recordings were especially successful as they can be used to interpret and aggregate findings across multiple events. ${ }^{17}$ Video recording of the same event or task at two levels of task urgency was particularly revealing as a means of identifying patient safety issues and systems failure and to perform ergonomic analyses. Because tasks such as emergency airway management or chest tube insertion are well circumscribed and occur frequently in trauma patients, there are many opportunities for video recording; the clinical staff can focus their attention on what aspect of care is under review; they can understand when video recording will start and finish; they can contribute their suggestions as SMEs providing "buy-in" to the project; and staff can understand the process for analysis and identify such events as breaches in safety performance by themselves and their colleagues when viewing video clips. Using this approach, our trauma centre clinicians have willingly reviewed video abstracts ( 15 second to 2 minutes long) copied onto compact discs (each of which holds up to 20 minutes of compressed video with adequate quality for review), and have reviewed these at times and locations chosen by the staff. During these abstract reviews the clinicians provide answers to specific questions linked to specific video abstracts. This approach allows completion of inter-rater reliability statistics for probing performance or safety aspects of the individual steps in the resuscitation of a trauma patient or of a studied task.

\section{Uses of video compared with observational data}

Applications of the video records we have reported include an examination of collaboration in teams ${ }^{18}$; comparison of performance of real video recorded events recreated in a full mission patient simulator ${ }^{19}$; occurrence of fixation errors and failure of standard operating procedures in real clinical practice $^{7}$; decision making in dynamic environments such as trauma patient resuscitation ${ }^{20}$; and monitoring of behavior in surgical operating rooms. ${ }^{21}$ We video recorded and identified visual scanning patterns using an eye tracker during remote diagnose ${ }^{22}$ and described metrics of uncertainty during video records of resuscitation of trauma patients. ${ }^{23}$ Components of task complexity in emergencies were captured on video records ${ }^{24}$ and experiences with video analysis for performance modelling were described..$^{15}$ An ergonomic analysis of the trauma resuscitation workplace using video was reported. ${ }^{9}$ Video analysis techniques and software are described at our website (http://hfrp.umm.edu/).

Video recording detected quality assurance occurrences not identified in self-reports such as the anaesthesia record or quality assurance reporting systems. ${ }^{25}$ Video records, unlike observational data, are a powerful feedback and training tool. Video data can be used in ways not originally envisaged when the record was made. Events or tasks not associated with the original analysis may be detected and data extracted later as, for example, with our analysis of vital signs monitor alarms in association with airway management ${ }^{26}$ which used existing records from our video library. Video clips can be used as stimulus material for individual training, distributed team training, examination of the impact of communication media on telemedicine decision making, and expertise coordination and collaboration.

\section{CONSENT AND CONFIDENTIALITY}

Protocols that included video recording were approved by the IRB for human subjects of research. Consent forms were signed by research subjects including anaesthesiology, surgeon, nurse and technician trauma care providers. Permission was obtained from the IRB to retain video abstracts of up to 2-3 minute duration for research and educational purposes provided the consent of the video recorded subjects was obtained. In these video abstracts all patient (and research subject, if requested) identifiers were removed by image blurring or by using specific camera angles or video segments that did not allow identification or recognition of individuals.

However, video is a very powerful medium. A concern is that, even when researchers obtain a subject's consent, it is not always clear that the subject understands the implications of that consent. We have used the process described in fig 5 as an extension of fulfilling the obligatory need to have a signed consent form. The consent process explains the implications to as many as possible of those affected by or likely to be included in the video recording. It is time consuming but rewarding because subjects understand what is happening.

It should be noted that fewer research subject participants are needed than might be thought to obtain video data. In our first project ${ }^{11}$ most of the 120 video recordings were made by just six research subjects. No consent forms were signed by other colleagues in the clinical workplace or the patient. 


\section{Pointers for future research}

- Video based research involving cognitive task analysis.

- Medical hardware and human interface design.

- Design principles for multi-specialty workplaces.

- Use of video to create a real life laboratory for studying team performance.

- Comprehensive and integrated video data acquisition and analysis tools.

- Establish test and validate video derived performance measurement instruments.

- Audio analysis tools and taxonomy of verbal communications.

- Video educational and training material preparation.

Instead, the consent process described in fig 5 for nonresearch subject clinicians was followed. During the first 3 years only two research subjects would not consent to participate or be included in video recordings. One additional subject agreed to participate only if their image was blurred in any retained video abstracts.

The IRB agreed to allow video recording without patient consent for research protocols because it was not thought feasible to obtain consent in the emergency circumstances in which the video records were made. However, every effort was made to preserve privacy by using camera angles and tight image border control to avoid recognition of individuals. Patient identifiers were removed from paperwork associated with video records. To preserve confidentiality, only care providers and researchers were given access to the video records which were kept secure under two sets of locks.

Technical approaches used to preserve confidentiality and maintain privacy of the video recorded individuals included video masking with blurring of the face and other distinguishing features. Voices can be disguised, but these digital manipulations can impair video data analysis if qualities of speech or gaze are being analyzed. The key to the consent process and confidentiality is, in our opinion, the development of trust by those who are videotaped that the investigators will not abuse the privilege of being allowed to acquire video data for research purposes.

Generally, the original video records were destroyed by degaussing within 4-6 weeks of collection. A sign at the entrance to the operating rooms and inside the trauma resuscitation unit was posted to indicate that image recording was occurring. The wording "Be aware, filming is underway" complies with Joint Commission of Accreditation of Hospital Organizations (JCAHO) regulations for video recording in hospitals. During 11 years of video recording we have experienced no medicolegal subpoenas and no employment related or liability issues.

We believe that this review of video data extraction and analysis techniques used in a clinical environment may help other researchers in formulating their research plans and in data analysis when using video recording. This belief is based on published reports on video analysis, ${ }^{561627-32}$ as well as comments, feedback and personal communications received from a wide variety of sources.

\section{DISCUSSION}

Video records are particularly revealing and have benefits over observation for analysis of critical events, trauma resuscitation, anaesthesia, and surgical tasks. They have a number of applications including performance feedback,

\section{Key messages}

- It is important to develop trust with video recorded subjects.

- Clinician feedback should be obtained on introduction of a new protocol or line of investigation.

- Aggregated video recorded data should be reported and clinician reviews used for feedback

- Task analysis at two levels of task urgency is powerful methodology for brief and risky but beneficial tasks.

- Multidisciplinary experts in surgery, anesthesiology, and nursing should be involved.

- Audio records of participants should be used to explain cognitive aspects of events or covert processes.

- Where events are uncertain or verbal interactions unclear, participant input is needed for clarification.

- Single critical events may reveal underlying systems failures.

- Video records detect quality assurance occurrences not identified by self-reports.

- Video provides powerful feedback and video clips are important training tools.

quality improvement, clinician training, as educational tools, and for human factors and ergonomic research.

Video recording is an underused data collection tool in clinical practice because of medicolegal, confidentiality, privacy, and employment performance related risks. This paper documents some of the benefits of a phased scale up strategy of video recording and the outcomes of 11 years of experience. While observation is the traditional approach to identifying problems and implementing improvements, this approach was unable to detect problems or to find solutions for correction, such as were found as a result of systematic analysis of video records.

\section{Actual and potential impact of video analysis}

Video analysis of a prolonged uncorrected oesophageal intubation resulted in the development of a task/communication algorithm used for trainees and in clinical practice by anaesthesia care providers. ${ }^{7}$ Implementation of this best practice for tracheal intubation has resulted in avoidance of uncorrected oesophageal intubation in the trauma centre for the last 9 years. Video analysis of chest tube insertion confirmed a published suggestion that the $16 \%$ incidence of empyema following tube thoracostomy at our trauma centre was a result of contamination during insertion. ${ }^{33}$ We found breaks in sterile technique in all 49 chest tube insertions video recorded in elective and emergency circumstances. We have instigated training in surgical techniques to avoid the identified problems. By suggestions for instrument tray positioning and revisions in the tray content and use, it is to be hoped that operator injury (two needle sticks and one knife cut in 49 video records) can be reduced with the revised chest tube insertion procedures. The redesigned nurses' supply cart in the trauma resuscitation unit has resulted in increased nurse satisfaction. Reconfiguration of the anaesthesia workspace, equipment, and monitor placement in the trauma resuscitation unit has simplified access for emergency resuscitation and patient monitoring equipment.

\section{ACKNOWLEDGEMENTS}

The work carried out in the Shock Trauma Centre and reported in this paper at the University of Maryland would not have been possible without the support and collaboration of the physicians, 
nurses, technicians, pre-hospital providers, and administrators who work there. The views expressed are those of the authors and do not represent the opinions of the funding agency.

\section{Authors' affiliations}

C F Mackenzie, Professor, Director, The Charles McC Mathias Jr, National Study Centre for Trauma and EMS at the University of Maryland School of Medicine, Baltimore, MD 21201, USA

Y Xiao, Associate Professor of Anesthesiology, Director, Human Factors Research Laboratories, Faculty, National Study Centre for Trauma and EMS at the University of Maryland School of Medicine, Baltimore, MD 21201, USA

Supported by AHRQ grants 5U18HS1 $1279-02$ and 5P2OHS1 1562-02.

\section{REFERENCES}

1 Seagull JF, Sanderson PM. Anesthesia alarms in context: an observational study. Human Factors $2001 ; 43: 66-78$.

2 Klein GA. A recognition primed decision (RPD) model of rapid decisionmaking. In: Klein G, Orasanu J, Calderwood R, Zsambok CS, eds. Decision making in action: models and methods. Norwood NJ: Ablex Publishing Corp, 1993: 138-47.

3 Roth EM, Patterson ES. Using observational study as a tool for discovery: uncovering cognitive and collaborative demands and adaptive strategies. Proceedings of 5th Naturalistic Decision Making Conference. Sweden, May 2000.

4 Woods DD. Process training methods for the study of cognition outside the experimental psychology laboratory. In: Klein GA, Orasanu J, Calderwood CE, Zsambok CS, eds. Decision-making in action: models and methods. Norwood NJ: Ablex Publishing Corp, 1993.

5 Mackay WE, Tatar DG. Introduction to special issue on video as a research and design tool. Special Interest Group on Computer and Human Interaction (SIGCHI) Bull 1989;21:48-129.

6 Nardi BA, Kuchinsky A, Whittaker S, et al. Video-as-data: technical and social aspects of a collaborative multimedia application. In: Finn KE, Sellen AJ, Wilbur SB, eds. Video-mediated communication. Mahwah, NJ: Erlbaum Associates, 1997:487-517.

7 Mackenzie CF, Martin P, Xiao Y, et al. Video analysis of prolonged uncorrected esophageal intubation. Anesthesiology 1996;84:1394-503.

8 Mackenzie CF, Xiao Y, Hu P, et al. Video clips as a data source for safety performance. Proceedings of Human Factors and Ergonomics Society 47th Annual Meeting, 2003 (in press).

9 Harper BD, Mackenzie CF, Norman KL. Quantitative measures in the ergonomic examination of the trauma resuscitation unit's anesthesia workspace. Proceedings of Human Factors and Ergonomics Society 47th Annual Meeting, 1995;2:723-7.

10 Seagull JF, Mackenzie CF, Bognerl SM. The hidden virtues of video analysis. Proceedings of Human Factors and Ergonomics Society 47th Annual Meeting, 2003 (in press).

11 Xiao Y, Mackenzie CF. Stress and decision-making in trauma patient resuscitation. Final report available at http://hfrp.umm.edu/onrrep.

12 Mackenzie CF, Xiao Y, Hu PF-M, et al. Remote decision-making: media, cues and diagnosis during dynamic tasks. Final report available at http:// hfrp.umm.edu/nasarep.
13 Mackenzie CF, Hu PFM, Xiao Y, et al. Video acquisition and audio system network (VAASNET) for analysis of workplace safety performance. Biomed Instr Tech 2003;37:285-91.

14 Annett J. Theoretical and pragmatic influences on task analysis methodology. In: Schraagen JM, Chipman SF, Shalin VL, eds. Cognitive task analysis. New Jersey: L Erlbaum, 2000:25-37.

15 Zachary WW, Ryder JM, Hicinbothom JM. Building cognitive task analyses and models of a decision-making team in a complex real-time environment. In: Schraagen JM, Chipman SF, Shalin VL, eds. Cognitive task analysis. New Jersey: L Erlbaum, 2000:365-83.

16 Harrison S, Minneman S, Stulls B, et al. Video: a design medium. Special Interest Group on Computer and Human Interaction (SIGCHI) Bulletin 1989:21:62-6.

17 Mackenzie CF, Xiao Y, LOTAS Group. Video analysis for performance modeling in real environments: methods and lessons learned. Proceedings of Human Factors and Ergonomics Society 43rd Annual Meeting 1999:237-41.

18 Xiao Y, Mackenzie CF. Collaborative crew performance in complex operational systems. NATO HFM Symposium, Edinburgh, UK 1998:4:4-11.

19 Mackenzie CF, Harper BD Xiao Y Simulator limitations and their effects on decision-making. Proceedings of Human Factors and Ergonomics Society 40th Annual Meeting 1996;11:747-51.

20 Xiao Y, Mackenzie CF, LOTAS Group. Decision-making in dynamic environments: fixation errors and their causes. Proceedings of Human Factors and Ergonomics Society 39th Annual Meeting 1995:469-73.

21 Seagull FJ, Xaio Y, Mackenzie CF, et al. Monitoring behavior: a pilot study using an ambulatory eye-tracker in surgical operating rooms. Proceedings of Human Factors and Ergonomics Society 43rd Annual Meeting 1999:850-4.

22 Xiao Y, Mackenzie CF, Orasanu J, et al. Visual scanning patterns during remote diagnosis. Proceedings of Human Factors and Ergonomics Society 42nd Annual Meeting 1998:222-76.

23 Xiao $Y$, Mackenzie CF. Uncertainty in trauma patient resuscitation. Proceedings of Human Factors and Ergonomics Society 41 st Annual Meeting 1997:68-171.

24 Xiao Y, Hunter WA, Mackenzie CF, et al. Task complexity in emergency medical care and the implication of team coordination. Human Factors 1996;38:636-45

25 Mackenzie CF, Jefferies NHJ, Hunter WA, et al. Comparison of self-reporting of deficiencies in airway management with video analyses of actual performance. Human Factors 1996;38:623-35.

26 Xiao Y, Mackenzie CF, Seagull JF, et al. Managing the monitors: analysis of alarm silencing activity during an anesthetic procedure. Proceedings of International Ergonomics Association Congress 2000;4:250-93.

27 Sanderson PM, Fisher C. Exploring sequential data analysis: foundation. Human-Computer Interaction (Special issue on exploratory sequential data analysis) 1994:251-317.

28 Heath C, Luft P, Sellen A. Reconfiguring media space: supporting collaborative work. In: Finn KE, Sellen AJ, Willbur SB, eds. Video-mediated communication. Mahwah NJ: Erlbaum Associates, 1997:323-47.

29 Dowrick PW, Biggs SJ. Using video: psychological and social applications. Chichester: Wiley, 1983.

30 Carbine DN, Finer NN, Knodel E, et al. Video recording as a means of evaluating neonatal resuscitation performance. Pediatrics 2000;106:654-8.

31 Birnbach DJ, Santos AC, Bourlier, et al. The effectiveness of video technology as an adjunct to teach and evaluate epidural anesthesia performance skills. Anesthesiology 2002:96:5-9.

32 Bryne AJ, Sellen AJ, Jones JG, et al. Effects of videotape feedback on anaesthetists' performance while managing simulated anaesthetic crises: a multicentre study. Anaesthesia 2002;57:176-9.

33 Caplan ES, Hoyt NJ, Rodriquez A, et al. Empyema occurring in multiply traumatized patients. J Trauma 1984;24:785-9. 